\title{
FUENTE DEL SABER DE LOS PROFESORES. ENTRE EL IDEARIO PEDAGÓGICO DE RABINDRANATH TAGORE Y EL PERFIL, PARÁMETROS E INDICADORES PARA DOCENTES Y TÉCNICOS DOCENTES DE EDUCACIÓN BÁSICA
}

\author{
Enrique Ibarra Aguirre* \\ Universidad Pedagógica del Estado de Sinaloa
}

\section{RESUMEN}

El propósito de este artículo consiste en examinar las teorías educativas de Rabindranath Tagore para conocer la fuente del saber pedagógico de los profesores, así como los vínculos entre dicho saber pedagógico y los plasmados en el perfil, parámetros e indicadores (PPI) para docentes y técnicos docentes de educación básica, propuesto por la Secretaría de Educación Pública de México. Es una investigación comparativa de carácter documental cuyos hallazgos revelan que Tagore privilegió el conocimiento práctico para el ejercicio docente, lo que difiere del PPI, dado que en éste se privilegia más el saber derivado de las ciencias de la educación en la práctica docente. Aún se requiere más exploración del pensamiento de Tagore para construir su modelo pedagógico. El PPI debe mantenerse en la mesa del debate y ajuste, e incorporar el perfil de educadores de migrantes y multigrado.

Palabras clave: conocimiento práctico, Rabindranath Tagore, formación docente.

\section{SOURCE OF KNOWLEDGE OF TEACHERS. BETWEEN THE PEDAGOGICAL IDEOLOGY OF RABINDRANATH TAGORE AND THE PROFILE, PARAMETERS AND INDICATORS FOR TEACHERS AND TECHNICAL TEACHERS OF BASIC EDUCATION}

\section{Abstract}

The purpose of this article is to examine the educational theories of Rabindranath Tagore to know the source of pedagogical knowledge of teachers, as well as the links between this pedagogical knowledge and those expressed in the Profile, Parameters and Indicators (PPI) for teachers and technicians Teachers of basic education, proposed by the Secretariat of Public Education of Mexico. It is a comparative research of a documentary nature whose findings reveal that Tagore privileged, the practical knowledge for the teaching exercise, which differs from the PPI, since in this one the knowledge derived from the educational sciences is more privileged in the teaching practice. Even more exploration of Tagore's thinking is required to build his pedagogical model. The PPI should remain at the table of debate and adjustment, and incorporate the profile of migrant and multigrade educators.

KEYwORDs: practical knowledge, Rabindranath Tagore, teacher training.

DOI: https://doi.org/10.25145/j.qurricul.2019.32.04

Revista Qurriculum, 32; junio 2019, pp. 63-79; ISSN: e-2530-8386 


\section{INTRODUCCIÓN}

Para que el educador pueda responder con sentido y acierto a su realidad profesional, debe tener y poner en movimiento un cúmulo de saberes inherentes a su profesión, que sin duda no emergen de manera espontánea; tienen un punto de inicio y se esperaría que experimenten un periodo de desarrollo y consolidación (Marcelo \& Valliant, 2010). No obstante todo lo dicho, aún no se ha logrado un acuerdo sobre el repertorio de saberes que los profesores deben poseer y tampoco sobre la naturaleza de los mismos (Clandinin \& Conelly, 1987; Elbaz, 1981; Porlán \& Martín, 1994; Shulman; 1987; Tardif, 2004). Sin embargo, ha sido posible percibir en las diferentes reflexiones vertidas en la literatura al respecto la convergencia sobre el valor atribuido al saber que emana de la experiencia profesional docente, el cual se convierte en conocimiento práctico que, como teorías para la acción, se encuentran en constante dinamismo.

Pero entrar en esa discusión supera nuestros esfuerzos, aunque se hace necesario reconocerla porque en esta comunicación hay un interés en responder a las siguientes preguntas: ¿cuál es la fuente del saber de los profesores que se sugiere desde el pensamiento pedagógico de Rabindranath Tagore?, ¿qué vínculos existen entre esas teorías educativas y las dimensiones del documento perfil, parámetros e indicadores (PPI) para docentes y técnicos docentes de educación básica?

Para responder a esos cuestionamientos, primero se presenta una revisión documental sobre el escasamente estudiado pensamiento pedagógico de Rabindranath Tagore y se exponen sus planteamientos teóricos sobre la fuente del saber docente, luego se construye un perfil docente de acuerdo a sus ideales en un formato de cinco dimensiones, similar al que se ha elaborado en el PPI, descrito líneas abajo, que al parecer se erige como la expresión de los conocimientos que debe poseer el profesorado en México para tener un desempeño profesional docente eficaz. Realizado ello, se colocan frente a frente ambos perfiles -el de Tagore y el del PPI-, de tal forma que ello nos permita reconocer la presencia del ideario tagoriano en dicho documento y establecer similitudes y/o diferencias entre ambos perfiles en cuanto a la fuente del saber de los profesores.

*E-mail: enrique.ibarra@upes.edu.mx. Es licenciado en Psicología, maestro en Educación y doctor en Educación por la Universidad Autónoma de Sinaloa. Es integrante de la Red Temática CONACYT de Investigación de Educación Rural y la Red Iberoamericana de Educación en Territorios Rurales. Es profesor investigador adscrito a la Universidad Pedagógica del Estado de Sinaloa. Sus intereses de investigación son el estudio de la construcción compleja de la teoría del yo de los sujetos escolares y su relación con los procesos educativos, la formación de profesores y la educación en contextos rurales. 


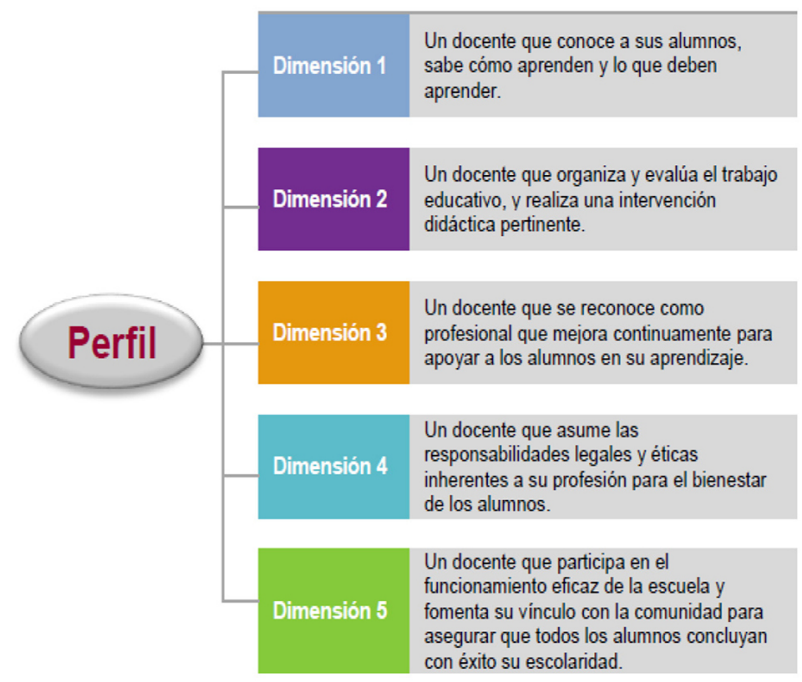

Figura 1. Perfil pentadimensional extraído del documento sobre perfil, parámetros e indicadores para docentes y técnicos docentes en educación básica (SEP, 2014, p. 12).

\section{UNA BREVE DESCRIPCIÓN DEL PPI}

No se pretende ser exhaustivo en los elementos contextuales en los que surge el PPI; no obstante, es importante destacar, primero, que nace en el marco de la muy debatida reforma educativa 2012-2013 en México, de la cual, recientemente se ha anunciado su despedida. Por otro lado, es importante decir sobre este documento (SEP, 2014) que en él se gesta una aproximación al holograma de la profesión docente que deben tener inscrito todos los educadores de educación básica en nuestro país, dado que en éste se definen

... las características, cualidades y aptitudes deseables que el personal docente y técnico docente requiere tener para un desempeño profesional eficaz, además de ser una guía que permita a maestras y maestros orientar su formación para desempeñar un determinado puesto o función en el sistema de Educación básica (p. 12).

También es significativo recuperar, tal como se lee en la cita anterior, que este documento debe guiar y orientar la formación de los profesores, y al instituirse como referente de la actuación docente, se constituye también en la lente desde la cual evaluar el desempeño de estos actores educativos, acción que recae en la Coordinación Nacional del Servicio Profesional Docente de la Secretaría de Educación Pública.

El documento del PPI posee una estructura pentadimensional jerárquica. El primer nivel está compuesto por los dominios fundamentales del desempeño docente de los profesores de educación básica (preescolar, primaria y secundaria) (véase figura 1). Y en el segundo nivel se perciben constituyentes más específicos, 
elaborados en forma de parámetros descriptivos más particulares del saber y quehacer docente, cada uno con sus respectivos indicadores, que aquí no se describen puntualmente, dado que ellos se han elaborado de forma diferenciada para cada nivel que compone la educación básica (SEP, 2014).

\section{EL MULTIFACÉTICO RABINDRANATH TAGORE (1861-1941)}

El lector debe saber que el pedagogo del cual se reflexiona aquí fue un personaje hindú nacido en el último tercio del siglo XIX, cuya historia familiar más cercana le brindó una atmósfera con ejemplos y estímulos que lo llevaron a conocer muy bien la poesía, el teatro, la música y la filosofía, entre otras disciplinas. Quizás a ello se debe en gran medida la expresión de sus facetas como escritor, filósofo, músico, pintor, dramaturgo y poeta. Esta última como la más conocida, y la que le valió el reconocimiento mundial para ser laureado con el Premio Nobel de Literatura en 1913, distinguiéndolo desde entonces como el poeta del mundo (Navdeep, 2010).

No obstante lo que se sabe y reconoce hoy acerca de él en el ámbito literario, escasamente se conoce sobre su labor como educador y fundador de más de una escuela (Shantiniketan, Instituto Sriniketan, Siksha-Satra Vishva-Bharati); menos aún se sabe y se ha difundido sobre su ideario pedagógico y lo que él pensaba en cuanto a la naturaleza del saber docente y sus aportaciones a la formación de los educadores. Lo poco que ahora se sabe en estas facetas no se debe al poco valor que tengan, sino quizás sea, como lo han considerado algunos autores (Jha, 1994; Paz, 2005), debido a la trascendencia y diseminación de sus obras literarias, que podrían estar eclipsando a aquéllas. Precisamente, de estos ámbitos de reflexión y actuación menos difundidos de Tagore de los cuales parte la reflexión que se hace en esta comunicación.

Antes de adentrarnos de lleno en el contenido del documento, es preciso anticipar al lector sobre las limitaciones que se tuvieron para elaborar este trabajo:

a) que la expresión del pensamiento pedagógico de Tagore se encuentra muy dispersa y poco nítida dentro de sus obras literarias, salvo en aquellos documentos especializados en el tema de la educación;

b) algunas ideas se plasman en trabajos de autores coincidentes a su pensamiento, de su equipo de colaboradores y de sus seguidores;

c) una cantidad importante de su obra permanece en el idioma hindi y,

d) que es difícil acceder a todo el acervo documental del autor, dado que muchas de sus obras se encuentran resguardadas físicamente en diversas bibliotecas alrededor del mundo. 


\section{LA EXPERIENCIA COMO FUENTE DEL SABER DOCENTE EN TAGORE}

Primero habremos de reconocer cuál es la naturaleza de donde emana el saber docente de Tagore, dado que ello permea todos sus planteamientos teóricos sobre la educación y la formación de los profesores, que se hacen evidentes en el perfil docente que aquí se propone.

De forma reiterada Tagore sugirió que sus propuestas educativas, en su origen, no adherían a un saber académico y disciplinar, o que éstas fueran producto de una nueva teoría o un método especial de enseñanza, tampoco emanaba de libros especializados en las ciencias de la educación, ni tenían en su origen una formación profesional docente, pues no hay registro que permita evidenciar que él asistió a una escuela formadora de maestros (Elmhirst, 1961; Pearson, 1917; Tagore, 1917; 1917b).

Tampoco tuvieron su origen en la interiorización mimética de la actuación de sus profesores como una fuente reconocida del saber-hacer docente (Porlán y Martín, 1994), pues de ellos advierte mayormente experiencias escolares de maltrato y represión (Tagore, 1917a), que finalmente, a sus 40 años de edad, actuaron como el detonante para tomar la decisión de fundar la escuela experimental de Shantiniketan -morada de paz- en 1901 y modelar, desde su propia perspectiva, en un inicio, qué trato deberían brindar a los niños aquéllos que están interesados en ser maestros de escuela (Tagore, 1917, p. 111).

En una conferencia en Ginebra dijo algo referente que se asocia con lo anterior (Tagore, 1921, citado por Paz, 2014):

... Fundé mí escuela hace veinte años, mas, a decir verdad, no tenía entonces método ni experiencia en la enseñanza [...] esto no es rigurosamente exacto. Tenía cuando menos, una experiencia negativa adquirida en el curso de mis propios años de escuela. Sabía cómo no deben ser tratados los niños.

De igual forma, sobre el Instituto experimental de Sriniketan, fundado años después, que tenía como propósito la reconstrucción de las comunidades rurales, Tagore también manifestó que tuvo origen en las memorias de su juventud sobre los grandes problemas que subyacían a los pueblos: económicos, de salud, educación, el deterioro de la identidad y pérdida de autonomía (Elmhirst, 1961), opuestas a su filosofía orientada a la libertad del hombre en todos los sentidos (Tagore, 1922; 1925).

La naturaleza de su saber surgió de otra fuente. Cuando Tagore puso en marcha Shantiniketan y luego Sriniketan, admitió que su saber-hacer fue muy incipiente, incierto y con poca nitidez; no tenía una ruta más o menos clara sobre cómo debería desempeñarse como maestro o de sus propuestas de cambio y mejora educativa, pero también admite que fueron tomando forma, claridad y madurez en la medida en que experimentaba con uno y otro sistema (Elmirst, 1961; Sen, 1943; Tagore, 1917).

De igual forma, el internado Siksha-Satra para nińos y nińas de las comunidades rurales, incorporado al Instituto Sriniketan, emanó de la experiencia educativa en Shantiniketan y dos años de desarrollo en Sriniketan, cuyos principios pedagógicos, como lo advierte Elmhirst (1961), son deducciones de sentido común, que 
devienen de los fracasos y éxitos en dichas instituciones. Su saber, al parecer, siguió la vía del saber intuitivo, ésa que siguen muchos profesores cuando lo que aprendieron en la fase de formación inicial no les ayuda mucho a dar respuesta a los incidentes críticos que se les presentan en su actuación docente día a día, por lo que recurren al ensayo y error (Porlán \& Martín, 1994).

Con base en la descripción que hace Tardif (2014), sobre los saberes del docente y su desarrollo profesional, en Tagore se advierte un saber experiencial, cuyo carácter práctico estuvo dado por la capacidad de apertura del pedagogo para reconocer y revisar los incidentes críticos que se le presentaban en su práctica docente, ir haciendo los ajustes necesarios e integrar las experiencias y conocimientos nuevos, los cuales ponían en dinamismo su persona y saber-hacer. Como él lo dijo en alguna ocasión, el crecimiento de su escuela -Shantiniketan- también significó el crecimiento de su vida (Tagore, 1917), en la cual, a partir de su práctica docente aprendió incluso la gramática, la aritmética y muchas cosas que había olvidado de su periodo formativo en la escuela (Tagore, 1921, citado por Paz, 2014).

De igual forma, habrá de reconocerse el carácter existencial en el saber-hacer de Tagore y en todo su pensamiento pedagógico (Tardif, 2014). Lleva indudablemente de suyo las huellas que dejaron su propia historia de vida, marcado en gran parte por las vivencias positivas dentro del seno familiar, mayormente por la influencia educativa de su padre, los numerosos viajes que realizó por todos los continentes y las instantáneas negativas que acumuló del sistema educativo de sus tiempos (Jha, 1994; Paz, 2014), pero también hay que reconocer que Tagore vivió un momento histórico marcado por un deseo vehemente por la libertad, evidente por el movimiento libertario que se inició en la India a mediados del siglo xIx y que concluyó a mediados del xx (Metcalf y Metcalf, T. 2014).

Todo ello le llevó a decidirse a ser maestro, no porque él se creyese un ser dotado con un talento especial para enseñar a los niños, sino porque con el cúmulo de experiencias vividas creía tener el secreto de hacerlos dichosos (Tagore, 1921, citado por Paz, 2014) y ponerlos en condición de ser libres de toda imposición externa. Hay que decir que la mayoría de sus producciones literarias y del ideario pedagógico de Tagore está impregnada por ese anhelo de libertad.

Es evidente que Tagore estaba convencido de la importancia que tiene el conocimiento práctico y la retroacción que debe realizar el profesor sobre su propio ejercicio docente, en el sentido de que pueda ponerse en circunstancia de realizar acomodaciones a su saber-hacer para hacerlo cada vez mejor, tal como más recientemente lo plantean Schön (1998) y Jacobo (2009) al referirse a la reflexión práctica como una dimensión que debe estar en la base del profesionalismo de los profesores.

El origen como maestro de escuela de nuestro pedagogo, como se dijo antes, se sustenta en gran parte en un ejercicio continuo de reflexión (Tagore, 1917), pero no niega en su desarrollo profesional la importancia de la búsqueda inteligente del saber técnico como otro componente indispensable para saber-hacer (Jacobo, 2009). Él mismo se abocó a la lectura de la pedagogía clásica y contemporánea, como líneas adelante se hace más explícito. Incluso Pearson (1917) se refería a la escuela experimental Shantiniketan como un híbrido entre las mejores tradiciones hindúes y los más sanos principios de los métodos modernos, particularmente aquéllos que 
ponían como baluarte a la libertad en toda su expresión y oponían franco rechazo a aquellos métodos represivos y los que privilegiaban la memorización como estrategia metodológico-didáctica.

Por último, es importante agregar que Tagore también creía en el poder del vínculo de la interacción con las experiencias y saberes de otros profesores como una fuente importante de saber-hacer. En algunas de sus obras literarias se deja ver la relación que mantenía con sus colegas, con los cuales compartía ideales educativos, experiencias docentes y amistad. De ellos reconocía su buen desempeño docente y dijo haber aprendido mucho (Pearson, 1917; Tagore, 1917).

De acuerdo a lo dicho hasta aquí, en el ejercicio docente de Tagore, en el sentido de la caracterización que realiza Tardif (2014) sobre el saber y desarrollo profesional docente, se privilegia el conocimiento práctico, el cual responde a un saber experiencial, con carácter heterogéneo, que se erige como una totalidad integrada de las múltiples experiencias como docente, de su historia de vida, pero también del reconocimiento de los saberes técnicos suficientemente probados, de la interacción con sus pares y todo lo que lleva de suyo como persona.

En los siguientes apartados, el lector encontrará algunas de las ideas pedagógicas sobre la naturaleza del saber de los profesores que emanan de ese conocimiento experiencial de Tagore, las cuales aquí nos sirven para elaborar un perfil de profesor siguiendo la estructura pentadimensional con que se elaboró el documento del PPI, con el fin de ir comparando uno a uno los planteamientos holográmicos de la profesión docente del pedagogo y los que se distinguen en el PPI.

\section{UN PROFESOR QUE CONOCE A SUS ALUMNOS. ENTRE EL SABER TÉCNICO Y EL PRÁCTICO}

Entre el componente del perfil del profesor enunciado en la dimensión uno del PPI para profesores de educación básica y el que se elabora con base en los ideales de Tagore, como se aprecia en la tabla 1, pareciera que no existe mucha diferencia, dado que en ambos se expresa un interés genuino por conocer todo cuanto sea posible de los educandos. No obstante, sí distan entre sí en la naturaleza del saber que suponen deben poseer los profesores para su actuación docente.

\begin{tabular}{ccc}
\hline \multicolumn{3}{c}{ TABLA 1. PERFIL DEL PPI Y TAGORE EN LA DIMENSIÓN UNO } \\
\hline Dimensión & \multicolumn{1}{c}{ PPI } & \multicolumn{1}{c}{ PERFIL DOCENTE EN TAGORE } \\
\hline \multirow{3}{*}{1} & $\begin{array}{l}\text { Un docente que conoce a } \\
\text { sus alumnos, sabe cómo } \\
\text { aprenden y lo que deben } \\
\text { aprender. }\end{array}$ & $\begin{array}{l}\text { Un docente que siempre está atento a sus alumnos para aprender } \\
\text { de conducirlos por la senda del saber y el conocimiento práctico } \\
\text { del mundo. }\end{array}$ \\
\hline
\end{tabular}

Nota: elaboración con base en el PPI y en el análisis documental de los textos de y sobre Tagore.

En esta dimensión del PPI se percibe que el conocimiento que debe poseer el docente, sin importar en qué nivel de educación básica labore, debe proceder más de un saber técnico, teniendo como el lente a las teorías del desarrollo y del apren- 
dizaje para conocer a sus alumnos y, para guiar su práctica, debe conocer los referentes pedagógicos y didácticos (SEP, 2014, pp. 22, 32, 42).

En Tagore, en cambio, la demanda de conocimiento por parte de los docentes está más identificada con el saber práctico, cuya naturaleza radica en la observación de la actividad diaria de los niños. El pedagogo decía que los profesores deben aprender a conocer qué les gusta y qué no les gusta a sus alumnos para poder discurrir entre ellos por la senda "del saber elevado» (Tagore, 1917a, p. 61).

Pearson (1917), destacado maestro en Shantiniketan que conocía y compartía los ideales de su amigo Tagore sobre la educación, decía que éste

proponía que los docentes conocieran el entorno en que se mueven y comprendieran todo cuanto sea posible de los niños, pues sólo mediante el conocimiento de ellos; sus características, su naturaleza, sus preferencias, su voluntad, entre otras cosas, podrían desarrollar sus capacidades profesionales y sólo así se puede estar al nivel de ellos y estimularlos de la forma más natural. La pauta de qué se debe enseñar partía de los intereses de los niños y no devenía del interés del profesor.

Tagore, estaba predominantemente más identificado con el conocimiento práctico; le asignó más valor al saber experiencial sobre los educandos como guía para educarlos que al saber técnico (Tagore, 1917; 1921).

En la conferencia dada en el Instituto Jean-Jacques Rousseau en Ginebra, Suiza (Tagore, 1921, citado por Paz, 2014), resumió de forma muy precisa sus ideales pedagógicos diciendo:

Se desprende de mis ideas, si gustáis, un solo principio director: ir a la vida allí donde reine. Salid de la sala de clases. No llevéis los árboles a la clase sino trasportad a la clase debajo de los arboles [...]. No os preocupéis de los métodos. Dejad que vuestro instinto os guíe hacia la vida. Difieren los niños unos de otros y es preciso aprender a conocerlos, navegar entre ellos como se navega entre escollos. Para aprender la geografía de sus espíritus es el mejor guía un espíritu misterioso que simpatiza con la vida.

Para puntualizar sobre la dimensión uno, hay que resumir que mientras en el PPI el conocimiento disciplinar de las ciencias de la educación debe servir como el cristal para saber cómo aprenden los niños, cuáles son sus procesos de desarrollo y el que da sustento a la actividad docente, en Tagore el conocer todo de los niños tiene su origen en la experiencia directa con éstos, que da forma y contenido a su práctica docente.

\section{LA ORGANIZACIÓN DEL TRABAJO EDUCATIVO. DOS MIRADAS COMPLEMENTARIAS Y ANTAGÓNICAS}

Las ideas pedagógicas de Tagore comparten el interés declarado en la dimensión dos del PPI y sus parámetros e indicadores, en tanto que en ambas se promueven la atención a las necesidades educativas de sus estudiantes, el respeto a la diver- 
sidad, el uso de estrategias y materiales didácticos para el logro de los propósitos educativos, la evaluación del logro cognoscitivo y la generación de ambientes propicios para el aprendizaje.

\begin{tabular}{ccc}
\hline & \multicolumn{2}{c}{ TABLA 2. PERFIL DEL PPI Y TAGORE EN LA DIMENSIÓN DOS } \\
\hline Dimensión & \multicolumn{1}{c}{ PPI } & \multicolumn{1}{c}{ PERFIL DOCENTE EN TAGORE } \\
\hline \multirow{2}{*}{2} & $\begin{array}{l}\text { Un docente que organiza y } \\
\text { evalúa el trabajo educativo, } \\
\text { y realiza una intervención } \\
\text { didáctica pertinente. }\end{array}$ & $\begin{array}{l}\text { Un docente que se vale más de la creatividad y el instinto para el } \\
\text { trabajo educativo que de los métodos. Utiliza todos los medios } \\
\text { posibles para ello y evalúa los aprendizajes de los alumnos en } \\
\text { un ambiente de confianza y libertad. }\end{array}$ \\
\hline
\end{tabular}

Nota: elaboración con base en el PPI y en el análisis documental de los textos de y sobre Tagore.

No obstante la similitud que pudiera apreciarse en las propuestas de perfil desde ambas miradas, descritas en la dimensión dos (ver tabla 2), se alcanzan a observar diferencias muy puntuales. Los parámetros estipulados en el PPI para maestros y técnicos docentes de educación básica señalan que el profesor debe organizar su intervención docente, desarrollar estrategias y construir ambientes para el aprendizaje y finalmente evaluar los logros cognoscitivos de los estudiantes, pero predominantemente esas actividades estarán delimitadas por los enfoques disciplinares de las asignaturas y con base en determinados aprendizajes esperados (SEP, 2014, pp. 23, 33, 43).

Tagore, por su parte, influido por su pensamiento de libertad, apelaba más a la creatividad e imaginación del profesor para elaborar estrategias para el aprendizaje. En el pedagogo, el «saber-hacer implica toda la frescura, espontaneidad y el arte de inventar nuevas formas y utilizar cualquier medio para mantener a los educandos ocupados y entretenidos» (Tagore, 1917a, p. 159). Su preocupación por los métodos se percibe menos rígida, las estrategias didácticas y la creación de ambientes de aprendizaje responden más a lo natural y al propio instinto del docente (Tagore, 1921). Decía que con la creatividad e imaginación del docente, hasta los más fútiles materiales son de mucho valor para los fines de la educación (Tagore, 1925, pp. 136137). El siguiente texto es ilustrativo de ello:

Para mí, la tabla de multiplicar está inscrita en los pétalos de las flores y en las nerviaciones de las hojas; sin saberlo, las mariposas la transportan en sus alas. Yo les he dicho esto a mis amigos los profesores de matemáticas, proponiéndoles que sacasen partido de ello en sus enseñanzas (Tagore, 1921, citado por Paz, 2014).

«Por otra parte, en Tagore no se percibe una obsesión por la evaluación de los niños para generar evidencia de que se alcanzan ciertos aprendizajes esperados o para obtener una credencial que sirva de evidencia de que se logran determinadas competencias, pues él aseguraba que esa práctica era más bien con fines del mercado laboral y no para satisfacer la naturaleza y desarrollo del ser humano» (Tagore, 1922, pp. 178-180). Pero sin duda, sí evaluaba, y cuando lo hacía generaba un ambiente de confianza y libertad en el que los estudiantes se quedaban solos resolviendo los ejercicios evaluativos, pues finalmente este ejercicio no conllevaba fines punitivos, sino 
constructivos, de tal forma que les sirviera a los profesores para conocer el estado de aprendizaje de los estudiantes y retroalimentarlos.

\section{EL DESARROLLO PROFESIONAL DOCENTE. UNA TAREA DE TODOS LOS DÍAS}

Es quizás la dimensión tres del PPI la que más similitud presenta con el pensamiento teórico de Tagore, en cuanto que en ambos hay un interés explícito por el desarrollo profesional de los profesores. En la tabla 3 se aprecia dicha dimensión y el perfil docente que se elabora con base en las teorías educativas de nuestro pedagogo.

\begin{tabular}{|c|c|c|}
\hline \multicolumn{3}{|c|}{ TABLA 3. PERFIL DEL PPI Y TAGORE EN LA DIMENSIÓN TRES } \\
\hline DiMENSIÓN & PPI & Perfil docente en Tagore \\
\hline 3 & $\begin{array}{l}\text { Un docente que se reconoce } \\
\text { como profesional que mejora } \\
\text { continuamente para apoyar a } \\
\text { los alumnos en su aprendizaje. }\end{array}$ & $\begin{array}{l}\text { Un docente que revisa su práctica y aprende de ella, sabe que } \\
\text { el conocimiento no está dado de una vez y para siempre, por } \\
\text { lo que actualiza sus saberes con el fin de estar en condiciones } \\
\text { de desatar procesos reales de aprendizaje en sus alumnos. }\end{array}$ \\
\hline
\end{tabular}

Nota: elaboración con base en el PPI y en el análisis documental de los textos de y sobre Tagore.

Explícitamente los indicadores del PPI en esta dimensión hacen énfasis en que el profesor identifique los aspectos de su práctica que requieren mejora, reflexione en ellos y se apoye en la teoría y en la discusión con sus pares para conseguir mejorarlos. De igual forma, para su desarrollo profesional docente sugiere que busque estratégicamente la información que requiera y realice las lecturas pertinentes para los fines educativos (SEP, 2014, pp. 24, 34, 44).

Para Tagore, igualmente, era muy importante que el profesor se comprometa con su propio desarrollo profesional; reconocía que ser maestro es una labor ardua y no muy fácil de lograr (Tagore, 1917, p. 134), y quien quiera serlo deberá entender que no basta la etapa formativa inicial para ello, dado que no se conoce de una vez y para siempre, sino que aprender es una tarea de toda la vida y deberá mantenerse en la senda del aprendizaje de continuo a fin de poder irradiar luz brillante y presentar nuevos contenidos a sus estudiantes.

Tagore fue un ejemplo de ello. Él fue un ávido lector de la pedagogía de su época; conocía, por ejemplo, las experiencias educativas de Pestalozzi y Froebel, el Emilio de Rousseau, y había leído al pedagogo John Dewey y a Herbert Spencer, conoció personalmente a Clapàrede y fue gran amigo de Pierre Bovet. Se sabe que llegó a documentarse sobre otras escuelas nuevas en Europa, las visitó y compartió correspondencia con sus fundadores, entre los cuales figuran Paul Geheeb, creador de una escuela nueva en Suiza y otra en Alemania. Estuvo en constante comunicación con María Montessori y la recibió en su escuela, cuyo método pedagógico Tagore recuperó y lo puso en fase experimental en algunas aulas en su escuela en Shantiniketan (Paz, 2013). Mientras hablaba con estudiantes de una escuela normal para maestros en Tokio les dijo: «... sé que algunos de los que me oyen están 
estudiando para maestros [...] para ser maestro de niños es completamente necesario ser como niños, olvidar lo que sabemos y que hemos llegado al término de los conocimientos» (Tagore, 1917b, p. 104).

Sabía que un maestro que no se actualiza, su estado más probable será la entropía cognitiva y profesional, por lo que lo único a realizar es repetir las mismas lecciones, llenar las mentes de los estudiantes con contenidos de pedagogías muertas, pero con pocas probabilidades de desatar en ellos procesos reales de aprendizaje (Tagore, 1922, p. 187).

El PPI y las ideas de Tagore en esta dimensión también presentan similitud en cuanto a que en el documento del PPI se alude a la importancia que tiene la participación colegiada de los profesores dentro del Consejo Técnico Escolar para su desarrollo profesional, lo cual es similar al Comité ejecutivo que tenía la escuela Shantiniketan de Tagore, en el cual el cuerpo de docentes se organizaba por asignaturas y discutían los materiales, libros y métodos de enseñanza (Pearson, 1917).

Otra afinidad entre el PPI y Tagore está en que el profesor sistematice y escriba sobre su práctica docente y la comparta con otros colegas. Compartir experiencias de la práctica que desarrollaban los profesores era común en Tagore y sus educadores; de hecho, él fundó la revista Visva-Barathi, fue el editor de ésta hasta su muerte, y en ella plasmó parte de sus ideas pedagógicas y algunos de sus profesores participaron narrando sus experiencias docentes.

\section{EL BIENESTAR INTEGRAL DE LOS ALUMNOS COMO FIN DE LA EDUCACIÓN}

De acuerdo con la tabla 4, en los primeros parámetros e indicadores de la dimensión cuatro, se lee un perfil de profesor que conoce los principios filosóficos del artículo tercero de la Constitución Mexicana y las disposiciones que rigen la labor del docente en México y con base en ellas conduce su ejercicio docente para el bienestar y desarrollo integral de los educandos (SEP, 2014, pp. 25, 35, 45).

\begin{tabular}{|c|c|c|}
\hline \multicolumn{3}{|c|}{ TABLA 4. PERFIL DEL PPI Y TAGORE EN LA DIMENSIÓN CUATRO } \\
\hline Dimensión & PPI & Perfil docente en Tagore \\
\hline 4 & $\begin{array}{l}\text { Un docente que asume las res- } \\
\text { ponsabilidades legales y éticas } \\
\text { inherentes a su profesión para } \\
\text { el bienestar de los alumnos. }\end{array}$ & $\begin{array}{l}\text { Un docente que se apega a las normas que rigen la operación } \\
\text { escolar, conoce los fines de la educación, promueve el respeto } \\
\text { por la diversidad, se conduce con sensibilidad y simpatía, genera } \\
\text { un ambiente de convivencia armónica y libertad que permitan } \\
\text { el bienestar, desarrollo de la creatividad y el aprendizaje de } \\
\text { los alumnos. }\end{array}$ \\
\hline
\end{tabular}

Nota: elaboración con base en el PPI y en el análisis documental de los textos de y sobre Tagore.

Tagore y los profesores de su escuela, en cambio, eran más autónomos, menos dependientes de reglamentaciones educativas externas; se regían por sus propias disposiciones normativas, tenían su propio currículum (Paz, 2013), pero 
también tenían como misión de la educación el logro de la integración de los aspectos físicos, intelectuales y espirituales de sus alumnos, la cual, decía, solo se logra a través de la senda de libertad, sin la mínima imposición externa (Tagore, 1917; 1922; 1925).

Se advierte en Tagore una postura sociocrítica contra el sistema educativo de sus tiempos, pues decía que ése servía a los intereses del gobierno y tenía una visión educativa prestada de Occidente, lo cual, lejos de procurar el bienestar y desarrollo integral de los individuos, por el contrario, regulaba y mutilaba el pensamiento y sentimiento, coartando la libertad y creatividad de profesores y de los estudiantes (Tagore, 1924, p. 78; 1928).

En otros parámetros e indicadores de esta dimensión hay mayor cercanía con los planteamientos de nuestro pedagogo, pues en ambos se busca no solo que el profesor reconozca y respete la diversidad, sino que ajuste su práctica docente con base en ello y que promueva la sana convivencia escolar. Tagore siempre fomentó el respeto por la diversidad y las diferencias de los otros en sus escuelas (Tagore, 1924, p. 61); de hecho, cuando fundó la Universidad Vishva-Barathi, fue con el propósito de unir Oriente y Occidente; sus estudiantes, con marcadas diferencias, tanto visibles (raza, idioma, religión) como invisibles (ideales, creencias religiosas), pudieran convivir en armonía y cooperación. En palabras poéticas Tagore pensaba a esta institución educativa como un espacio donde el mundo hace su hogar en un solo nido (Tagore, 1922).

La similitud también se observa cuando desde el PPI, en esta misma dimensión, se señala que el profesor debe saber que su actuar docente tiene consecuencias para sus alumnos. Rememorando sus vivencias escolares, decía que el profesor que no tiene el amor para enseñar a los niños o la aptitud para ser maestro, sería mejor que se emprendiera otra profesión porque algunos profesores, cuyo comportamiento era similar al de un tirano, pueden originar «los más grandes prejuicios a la Humanidad (...) y por esa aberración los niños sufren» (Tagore, 1925, p. 136).

\section{LA CONVIVENCIA ARMÓNICA COMO VÍA DEL DESARROLLO EDUCATIVO}

En lo general, entre el pensamiento tagoriano y la dimensión cinco del PPI (SEP, 2014, pp. 26, 36, 46) se aprecian puntos coincidentes (ver tabla 5). De manera particular, por ejemplo, en el primer parámetro, que refiere a la gestión escolar, tiene su parecido con la escuela de Tagore en cuanto que los profesores, como antes se dijo, se sentaban en sesión colegiada en calidad de Comité ejecutivo, a veces para atender la parte administrativa de la escuela y otras, para discutir los materiales de las distintas asignaturas y los métodos (Pearson, 1917).

Con respecto al cuidado que se debe hacer de los espacios, mobiliario y materiales escolares sugeridos por el PPI, no hay mucho que comparar con los planteamientos de Tagore, pues estas particularidades no representaban mucha importancia en nuestro pedagogo para cumplir con la misión de la educación; más bien, él prefería una vida más modesta en su escuela de Shantiniketan (Pearson, 1917), en 
la que el espacio no estaba limitado al encierro entre cuatro paredes dentro de las aulas. Los materiales de enseñanza, por otro lado, los podía encontrar sin costo en la naturaleza, debajo de los árboles, en el canto de los pájaros (Tagore, 1917) o en el aleteo de las mariposas (Tagore, 1921).

\begin{tabular}{|c|c|c|}
\hline Dimensión & PPI & Perfil docente en Tagore \\
\hline 5 & $\begin{array}{l}\text { Un docente que participa en el fun- } \\
\text { cionamiento eficaz de la escuela y } \\
\text { fomenta su vínculo con la comunidad } \\
\text { para asegurar que todos los alumnos } \\
\text { concluyan con éxito su escolaridad. }\end{array}$ & $\begin{array}{l}\text { Un docente que participa en la vida colegiada y admi- } \\
\text { nistrativa de la escuela, aprovecha del entorno aquellos } \\
\text { elementos que faciliten el aprendizaje de sus estudiantes } \\
\text { y se compromete con el desarrollo de las comunidades } \\
\text { y las personas. }\end{array}$ \\
\hline
\end{tabular}

Nota: elaboración con base en el PPI y en el análisis documental de los textos de y sobre Tagore.

Los indicadores del segundo parámetro de esta dimensión no están lejanos de las acciones de Tagore en su escuela, sobre todo el que refiere a establecer vínculos con otras instituciones para favorecer la tarea educativa, que gracias a ello, la escuela Shantiniketan recibía constantes presentes y apoyos económicos (Tagore, 1917), pero primordialmente, como antes se expuso, él buscaba relaciones académicas que fortalecieran sus ideales educativos para el bienestar de los estudiantes.

El tercer parámetro de esta dimensión del PPI, que alude a considerar los rasgos culturales y lingüísticos de la comunidad con el fin de favorecer el aprecio a la diversidad desde la escuela, es indiscutiblemente un ideal también explícito en Tagore, pues de forma reiterada, como ya se mencionó, insistía en que la escuela era el espacio para abolir las diferencias, donde se reconozca al otro (Tagore, 1922). Véase por ejemplo que en su escuela asistían estudiantes de castas diferentes a quienes se respetaban sus creencias (Pearson, 1917). Además, promulgó el cuidado de la cultura y la lengua de su tierra, sin dejar de reconocer, por supuesto, la riqueza y valor de otras culturas (Tagore, 1917).

\section{ALGUNAS CONSIDERACIONES FINALES}

Habrá de reconocerse, primero, que Tagore no recibió una formación inicial como la que recibe el profesorado actual en las Instituciones Formadoras y Actualizadoras de Docentes (IFAD); más bien, la naturaleza del saber de Tagore y lo que dio forma y contenido a su ideario pedagógico y praxis docente fue el conocimiento práctico; una síntesis dinámica que derivó de su experiencia profesional a partir de la reflexión sobre su propia práctica, de la observación de la actividad de sus educadores y educandos, de su repertorio existencial, de la interacción con sus colegas, de la construcción y recuperación de los métodos más apropiados y la lectura inteligente de la teoría para apoyar su práctica docente.

El profesorado de hoy, en contraste con la historia formativa de Tagore, tiene la ventaja de vivir procesos de formación más integral en alguna IFAD, pero 
la propuesta del autor acerca del compromiso con el conocimiento práctico como fuente de saber debe servir como un modelo para el profesorado de nuestros días. Dicho conocimiento será tan dinámico como sea su capacidad de ponerlos en movimiento; de reconocer que lo que sabe no está dado de una vez y para siempre y que es un saber cambiante a lo largo del ciclo profesional docente, por lo que permanentemente deberán mantenerse en la senda del saber, de tal modo que su conocimiento siempre sea vigente, atrayente e innovador para que pueda desatar verdaderos procesos de aprendizaje en sus estudiantes.

El ideario de Tagore debe invitar a todos los profesores a convertirse en ávidos observadores de su praxis; hacer de la reflexión sobre su práctica su más íntimo aliado para mejorarla y resolver los incidentes críticos que se le presenten en su práctica docente diaria. Asimismo, ser oportunistas en el sentido de valerse de todo cuanto haya a su alcance para que, con imaginación, invente nuevos modos para acompañar a los educandos en su trayecto formativo dentro de las instituciones educativas.

Otra consideración que se puede recuperar es que, entre el perfil para docentes y técnicos docentes de educación básica, recientemente propuesto en el documento del PPI, y el pensamiento tagoriano sobre la educación, que data de finales del siglo XIX, se pueden encontrar grandes similitudes. Cuando se encuentran diferencias, mayormente se expresan en la naturaleza del conocimiento de los profesores para realizar bien su saber-hacer. En el PPI se privilegia más el saber técnico derivado de las ciencias de la educación que el conocimiento práctico. En Tagore, en cambio, es este último el que predominantemente debe guiar todas sus estrategias didácticas de su práctica docente, aunque hay que reiterar que el pedagogo también le adjudica un valor importante al saber técnico, como un saber que debe ser complementario al conocimiento práctico.

Es importante destacar que el documento del PPI, en el sentido de ser una propuesta del holograma de saberes que los profesores de educación básica deben tener tatuados, como una especie de mapa que los conduzca durante la realización de su práctica docente, debería mantenerse en la mesa del diálogo entre expertos de las diferentes disciplinas de las ciencias de la educación, en constante retroalimentación por todos los actores educativos - principalmente los profesores- y en permanente actualización. Pero, además, sería importante que pudiera extenderse a otros escenarios educativos como en la educación para niños, niñas y adolescentes migrantes y las escuelas de organización multigrado en sus diferentes modalidades, donde las características del perfil docente que se demanda son diferentes.

Hay que señalar que Rabindranath Tagore es una veta del pensamiento oriental del cual apenas se han extraído de forma muy limitada algunas joyas pedagógicas con mucha vigencia en nuestros días. Algunos valores en materia de educación de su ideario aún siguen esperando aflorar y otros merecen mayor reflexión para empezar a construir el modelo pedagógico que subyace a sus planteamientos sobre la educación, en el que de forma integral se incluya lo que él pensaba sobre cómo aprenden y se desarrollan los individuos.

En el mismo sentido, deben revisarse con más profundidad los ideales que él tenía sobre el desarrollo de las comunidades rurales con base en sus planteamientos 
pedagógicos en la escuela experimental de Shantiniketan y Sriniketan, para atender a los grupos sociales más vulnerables. De igual forma conviene explorar lo que él pensaba sobre la formación de los profesores rurales. No obstante, con lo poco que se sabe y se ha divulgado sobre su ideario, se abre una puerta a un mundo nuevo de oportunidades educativas.

Recibido: 16-04-2019. Aceptado: 24-05-2019 


\section{REFERENCIAS BIBLIOGRÁFICAS}

Clandinin, D. y Connelly, F. (1987). «Teachers' personal Knowledge: What counts as 'personal' in studies of the personal». Journal of Curriculum Studies, 19(6), 487-500. (15/11/2018): DOI: https://doi.org/10.1080/0022027870190602.

Elbaz, F. (1981). «The teacher's practical knowledge: Report of a case study». Curriculum Inquiry, 11(1), 43-71. (15/11/2018): DOI: https://doi.org/10.1080/03626784.1981.11075237.

Elmhirst, L.K. (1961). «The foundation of Sriniketan», en Rabindranath Tagore y L.K. Elmhirst (eds.). Rabindranath Tagore: Pioneer in education. Essays and exchanges between Rabindranath Tagore y L.K. Elmhirst. London: John Murray.

JHA, N. (1994). «Rabindranath Tagore 1861-1941». Prospects: the quarterly review of education, 24(3/4), 603-619. (12/11/2018): DOI: http://www.ibe.unesco.org/sites/default/files/tagoree.PDF.

Jacoво-García, H.M. (2009). El profesionalismo integrado. Un nuevo modo de ser educador. México: UPN/Plaza y Valdés.

Marcelo, C. y Valliant, D. (2010). Desarrollo profesional docente. ¿Cómo se aprende a enseñar? Madrid, España: Narcea, S.A. de Ediciones.

Metcalf, B. y Metcalf, T. (2014). Historia de la India (3. ${ }^{\text {ra }}$ ed.). Madrid: Akal.

Navdeep, S. (2010). «Rabindranath Tagore 1861-1941». India Perspectivas, 24(2), 1.

PAz, R.J. (2014). «Grandes de la educación. Robindronath Tagore 1861-1941». Padres y maestros, 359. (12/11/2018): DOI: https://doi.org/10.14422/pym.i359.y2014.010.

PAz, R.J. (2013). «La Santiniketon de Tagore. La primera escuela nueva de Asia». Educar (NOS), 61, 3-6.

Pearson, W.W. (1917). Shantiniketan. The Bolpur School of Rabindranath Tagore. London: Macmillan and Co., Limited.

Porlán, R. y Martín, J.M. (1994). «El saber práctico de los profesores especialistas: Aportaciones desde las didácticas específicas». Investigación en la Escuela, 24, 49-58.

SEN, S. (1943). Rabindranath Tagore on rural reconstruction. Calcuta: Visva Bharati. (20.11.18): https:// archive.org/stream/in.ernet.dli.2015.247662/2015.247662.Rabindranath-Tagore_djvu.txt.

SEP. (2014). Perfiles, Parámetros e Indicadores para Docentes y Técnicos Docentes en Educación Básica. México: SEP.

SснӧN, D. (1998). El profesional reflexivo. Cómo piensan los profesores cuando actúan. España: Paidós.

Shulman, L. (1987). «Knowledge and Teaching: Foundations of the New Reform». Harvard Educational Review, 57(1), 1-21. (8/01/2019): DOI: https://doi.org/10.17763/haer.57.1. j463w79r56455411.

TAgore, R. (1917). Personality. London: Macmillan and Co., Limited.

Tagore, R. (1917a). My reminiscences. New York: The Macmillan Company.

TAgore, R. (1917b). «Paradise: being an address delivered by Rabindranath Tagore before Japanese students in Tokyo», en Pearson, W.W (ed.). Shantiniketan. The Bolpur School of Rabindranath Tagore. London: Macmillan and Co., Limited.

TAgore, R. (1921). "Conferencia en el Instituto Juan Jacobo Rousseau de Ginebra», editada por Pierre Bovet, ha sido publicada en la revista L'Educateur.

TAgore, R. (1922). Creative Unity. London: MacMillan and Co., Limited. 
Tagore, R. (1924). Nacionalismo. México: SEP/Universidad Nacional de México.

TAgore, R. (1925). «El maestro de escuela». Monitor de la educación común, 629, 135-137. Buenos Aires: Consejo Nacional de Educación. (10/11/2018): http://www.bnm.me.gov.ar/ ebooks/reader/reader.php?dir=00150891\&num_img=00150891_000000\&mon=1\&vn=s\&$\mathrm{vi}=s \& v \mathrm{t}=\mathrm{n} \& \mathrm{vp}=\mathrm{s} \& \mathrm{vv}=\mathrm{s} \& \mathrm{vh}=s \& \operatorname{modo}=\& z o o m=100 \& \mathrm{c}=\&$ buscar $=\& i \mathrm{r}_{-} \mathrm{a}=135 \& \mathrm{bus}-$ car $=I r+a+p \% E 1 g$.

TAGore, R. (1928). La escuela del papagayo y alocuciones en Shantiniketan. Barcelona: Editorial Cervantes.

TARDIF, M. (2004). Los saberes del docente y su desarrollo profesional. Madrid: Narcea. 
